\title{
The Evolving Role of Short-Term Professional Continuous Glucose Monitoring on Glycemic Control and Hypoglycemia Among Saudi Patients with Type 1 Diabetes: A Prospective Study
}

\author{
Ayman A. Al Hayek - Asirvatham A. Robert • Mohammed Al Dawish - Rania A. Ahmed • \\ Fahad S. Al Sabaan
}

To view enhanced content go to www.diabetestherapy-open.com

Received: June 7, 2015 / Published online: July 5, 2015

(C) The Author(s) 2015. This article is published with open access at Springerlink.com

\section{ABSTRACT}

Introduction: The aim of this study was to find out the evolving role of professional continuous glucose monitoring (PCGM) on hemoglobin A1c (HbA1c) and the frequency of hypoglycemia.

Methods: This was a 3-month, prospective study conducted among patients (aged 15-24 years) with type 1 diabetes mellitus who attended a diabetes clinic [Diabetes Treatment Center, Prince Sultan Military Medical City (PSMMC), Riyadh, Saudi Arabia] due to recurrent unexplained hypoglycemia unawareness episodes between July 2014 and December 2014. The respondents were purposively and conveniently selected and

Electronic supplementary material The online version of this article (doi:10.1007/s13300-015-0120-4) contains supplementary material, which is available to authorized users.

A. A. Al Hayek (囚) - A. A. Robert - M. Al Dawish · R. A. Ahmed · F. S. Al Sabaan

Department of Endocrinology and Diabetes,

Diabetes Treatment Center, Prince Sultan Military

Medical City, Riyadh, Saudi Arabia

e-mail: ayman.alhayek@yahoo.com they were asked to wear the PCGM device (iPro ${ }^{\circledR} 2$; Medtronic MiniMed, Inc., Northridge, CA, USA) for 5 days. The PCGM results were collected by the diabetic educator and reviewed by the treating physician on the same day as removal of the device. Clinical and demographic data were also collected.

Results: Overall, 56 patients were included in the study. The mean $( \pm \mathrm{SD})$ age of the study cohort was $18.1 \pm 1.82$ years and 27 (48.2\%) patients were male. Compared with baseline, non-significant but positive differences were observed in HbA1c levels in both male and female patients and in those who were older (aged 20-24 years). Similar results were observed in the frequency of hypoglycemia and a significant change was observed for female patients $(P<0.05)$. Compared with baseline, a significant positive difference was observed in patients' overall frequency of hypoglycemia by the end of the study $(P<0.001)$.

Conclusions: Professional continuous glucose monitoring is a valuable tool for detecting episodes of hypoglycemia and may help to decrease HbA1c levels and reduce the frequency of hypoglycemia. 
Keywords: Glycemic control; Hypoglycemia; Professional continuous glucose monitoring; Type 1 diabetes mellitus

\section{INTRODUCTION}

The latest International Diabetes Federation statistics suggest that Saudi Arabia has one of the highest percentages of diabetes sufferers in the world, which in turn means an increase in the number of diabetes-related complications $[1,2]$. Studies report that almost 1 in 4 people over 30 years of age has diabetes and that healthcare expenses incurred by people with diabetes have risen more than $500 \%$ in the last 18 years $[3,4]$. Furthermore, poor diabetes self-care management and a low adherence to medication are high among the Saudi population [5].

The current goal in diabetes management is to lower hemoglobin A1c (HbA1c), to target levels and to prevent diabetes-related complications while avoiding hypoglycemia [6]. Patients with diabetes are asked to reduce their HbA1c levels; as such, an increased need for more frequent and comprehensive glucose information with better hypoglycemia detection has led to the development of continuous glucose monitoring (CGM) technology [6].

Today, there are a large number of technologies available to assist with diabetes care. To maximize the benefits of this technology, it is essential that both clinicians and patients are involved. Recent clinical practice guidelines by the Endocrine Society suggest that patients aged 8 years and above who are offered CGM can benefit from this new technology $[7,8]$. Professional continuous glucose monitoring (PCGM) technology supports clinicians in gaining a more complete insight into their patient's glycemic profiles, variations in their lifestyle or dietary intake, and their therapeutic interventions [9]. Over the past 10 years, PCGM has also evolved from being a research tool to being a device that is useful in daily clinical practice [10]. Designed to improve glucose control without the addition of medication, CGM may have the potential to increase the proportion of patients who are able to maintain target HbA1c values while simultaneously limiting the risk of severe hypoglycemia $[7,8$, 10-12]. Also, research shows that the use of rapid and long-acting insulin analogs, improvements in insulin pumps, and repeated home blood glucose monitoring have had a positive effect on the ability of patients with type 1 diabetes mellitus (T1DM) to achieve target HbA1c levels [11]. However, rates of severe hypoglycemia remain too high and the occurrence of such events is often followed by a decline in glycemic control due to fears of further hypoglycemic episodes [11, 13]. This may also be due to circadian glycemic fluctuations and partially due to the lack of continuous feedback information on glucose levels after meals or during the night, although follow-up studies report a decreasing incidence [11, 13]. As a result, this represents the most significant barrier to achieving HbA1c target levels, in addition to its deleterious effects on the health and well-being of affected patients $[11,13]$.

It has never been easier to discover earlier the undetected high and low glucose excursions and to understand their causes. This data is supportive for making better treatment decisions to improve patients' lives. The iPro ${ }^{\circledR} 2$ (Medtronic MiniMed, Inc., Northridge, CA, USA) PCGM device is quick to implement and provides reports that physicians can use to educate patients on the therapy and lifestyle changes they can make to improve their health and quality of life. In the present study, the authors aimed to find out the impact of PCGM 
on patients HbA1c levels and the frequency of hypoglycemia.

\section{METHODS}

\section{Study Design and Setting}

This was a 3-month, prospective study conducted among patients (aged 15-24 years) with T1DM at the Diabetes Treatment Center, Prince Sultan Military Medical City (PSMMC), Riyadh, Saudi Arabia between July 2014 and December 2014. All patients had a diagnosis of T1DM according to the practice guidelines of the American Diabetes Association (ADA) [14].

\section{Patients Selection and Compliance with Ethics Guidelines}

Participants were selected according to their availability during their visit to the diabetes clinic. This study was conducted in accordance with the Helsinki Declaration of 1964, as revised in 2013, and the protocol of this study was approved by the research ethics committee of the PSMMC. All the patients were informed about the purpose and methods of the research both verbally and in written form. Written consent was obtained from patients before the completion of study measurement. Study participants were free to withdraw from the study at any time without giving a reason. During the baseline visit, socio-demographic, clinical data and patients treatment types, i.e., multiple daily injections (MDI) and insulin pump (IP) were collected.

\section{Inclusion and Exclusion Criteria}

Patients aged 15-24 years who had been followed up for at least 6 months and who did not have any other concomitant chronic disease were included. Exclusion criteria were adolescents who had a history of psychopathological and medical instability or visual, hearing, or cognitive impairment.

\section{Glycosylated Hemoglobin and Hypoglycemia}

The HbA1c test was the most appropriate measure of glycemic control and diagnostic test for diabetes. HbA1c $<7 \%$ was considered a good control value (ADA standards of medical care in diabetes) [14]. HbA1c levels were measured at baseline and 3 months. Readings were collected from the patients' records which are analyzed in the central lab at PSMMC (COBAS INTEGRA 400 plus/800 analyzers). Reports for PCGM were generated quickly within 1 day of removal of the device to identify patterns and to make the correct therapy adjustments by the same investigator.

Hypoglycemia was defined as any blood glucose value $\leq 70 \mathrm{mg} / \mathrm{dL}(\leq 3.9 \mathrm{mmol} / \mathrm{L})$. The frequency of hypoglycemia data was collected from patients' PCGM report [15].

\section{iPro2 and Measures}

A continuous glucose monitor is a minimally invasive device that is designed to measure and record glucose levels continuously and automatically in patients with diabetes mellitus [16]. The PCGM equipment is owned by the healthcare professional and is typically worn by the patient for 5 days. With PCGM, the patient remains unaware of blood glucose monitoring results until they are downloaded and analyzed by the healthcare professional with no alarms or inconvenience and requires minimum patient training when compared with real-time CGM, and patients do not 
receive glucose alerts and only see the PCGM data after it has been analyzed by the healthcare professional.

The iPro2 device was used over a 5-day period in a blinded fashion exclusively for CGM in all patients. The iPro2 was placed on and removed from the patient by a trained diabetic educator and all patients were advised to do four finger stick tests per day for calibration. The information was delivered to the treating physician within a day of removal of the device. The patients were educated to do routine self-monitoring of blood glucose (SMBG) and to record their dietary intake and activity during the test period. The test was ideally done on days that were representative of "typical" days. The results of iPro2 were discussed with the patients/care givers to make modifications concerning various factors influencing the patient's diabetes control, adjustments in insulin doses, and therapeutic treatment recommendations, including changes in diet, activity, and medication.

\section{Statistical Analysis}

Data analysis was performed using Excel 2010 (Microsoft Corporation, Seattle, WA, USA) and SPSS version 16 (SPSS Inc., Chicago, IL, USA). In addition to descriptive analysis, $t$ tests were used to look for differences between the groups and between baseline to 3 months. A $P$ value of $<0.05$ was considered statistically significant.

\section{RESULTS}

Overall, 56 patients were enrolled in the study. The mean $( \pm \mathrm{SD})$ age of the study cohort was $18.1 \pm 1.82$ years. Twenty-seven patients were male (48.2\%) and 29 were female (51.8\%; Table 1). More than $75 \%$ of the study population were receiving MDI treatment.
Table 1 shows the impact of PCGM on HbA1c and frequency of hypoglycemia. Compared with baseline, non-significant but positive differences were observed in HbA1c levels at 3 months in both male and female patients. Similarly, non-significant but positive differences were observed in patients aged 15-19 years and patients aged 20-24 years. Both treatment groups (MDI, IP) showed positive differences at 3 months compared with baseline. However, no significant differences in HbA1c levels were observed between genders, age groups, and different treatments groups.

Positive reductions were observed in the frequency of hypoglycemia at 3 months in both male and female patients when compared with baseline. Similar results were observed in the different age groups and the different treatment groups. It is important to note here that, when compared with male patients, females showed a significant reduction in the frequency of hypoglycemia $(P<0.05)$. However, no significant differences were observed between the different age groups and the different treatments groups (Table 1).

The impact of PCGM on overall HbA1c level is illustrated in Fig. 1. Compared with baseline, an non-significant but positive difference (0.42\%, $P=0.073$ ) was observed in HbA1c levels at 3 months. Compared to baseline, a positive significant difference was observed in the overall frequency of hypoglycemia at 3 months $(P<0.001)$; Fig. 2 .

\section{DISCUSSION}

People with diabetes face a life-long optimization problem to continue glycemic control without increasing their risk of hypoglycemia [17]. The developing trends in 
Table 1 The impact of professional continuous glucose monitoring on HbAlc levels and the frequency of hypoglycemia

\begin{tabular}{|c|c|c|c|c|c|}
\hline \multirow[t]{2}{*}{ Variables } & \multirow[t]{2}{*}{$n, \%$} & \multicolumn{2}{|c|}{ HbA1c level, \% } & \multicolumn{2}{|c|}{ Frequency of hypoglycemia, $n$} \\
\hline & & Baseline & 3 months & Baseline & 3 months \\
\hline \multicolumn{6}{|l|}{ Gender } \\
\hline Male & $27(48.2 \%)$ & $8.61 \pm 0.49$ & $8.36 \pm 0.23$ & $2.56 \pm 0.69$ & $2.04 \pm 0.19$ \\
\hline Female & $29(51.8 \%)$ & $8.95 \pm 0.66$ & $8.47 \pm 0.54$ & $2.63 \pm 0.5$ & $1.6 \pm 0.5^{*}$ \\
\hline \multicolumn{6}{|l|}{ Age, years } \\
\hline $15-19$ & $35(62.5 \%)$ & $8.91 \pm 0.63$ & $8.51 \pm 0.42$ & $2.59 \pm 0.56$ & $1.93 \pm 0.25$ \\
\hline $20-24$ & $21(37.5 \%)$ & $8.68 \pm 0.47$ & $8.31 \pm 0.41$ & $2.56 \pm 0.72$ & $1.72 \pm 0.57$ \\
\hline \multicolumn{6}{|c|}{ Treatment type } \\
\hline MDI & $44(78.6 \%)$ & $8.76 \pm 0.57$ & $8.57 \pm 0.42$ & $2.69 \pm 0.57$ & $1.89 \pm 0.31$ \\
\hline IP & $12(21.4 \%)$ & $8.85 \pm 0.62$ & $8.12 \pm 0.43$ & $2.18 \pm 0.62$ & $1.73 \pm 0.64$ \\
\hline
\end{tabular}

Values are presented as mean \pm standard deviation. Groups were compared by $t$ test

$H b A 1 c$ hemoglobin A1c, IP insulin pump, $M D I$ multiple daily injections

${ }^{*} P<0.05$, male vs. female

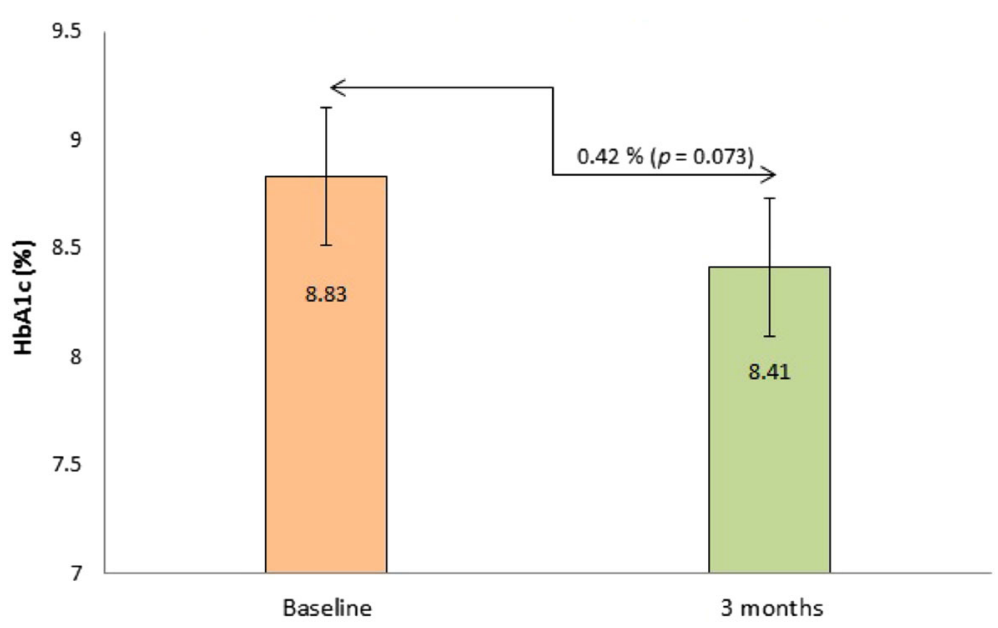

Fig. 1 The impact of professional continuous glucose monitoring on HbAlc levels. Values are presented as mean \pm standard deviation. Baseline vs. 3 months compared by $t$ test. HbAlc hemoglobin Alc

technology and therapeutic strategies create a very exciting time for the management of diabetes [18]. PCGM, being relatively novel technology, has been shown to be helpful and offers the potential to improve care for patients with T1DM beyond what can be achieved with SMBG alone [19]. In the present study, the authors aimed to discover the evolving role of
PCGM on HbA1c levels and the frequency of hypoglycemia. It was found that, when compared with baseline, positive (but nonsignificant) differences were observed in HbA1c levels among genders, different age groups, and different treatment groups. The overall HbA1c levels also showed positive decline. In contrast to our findings, in a 


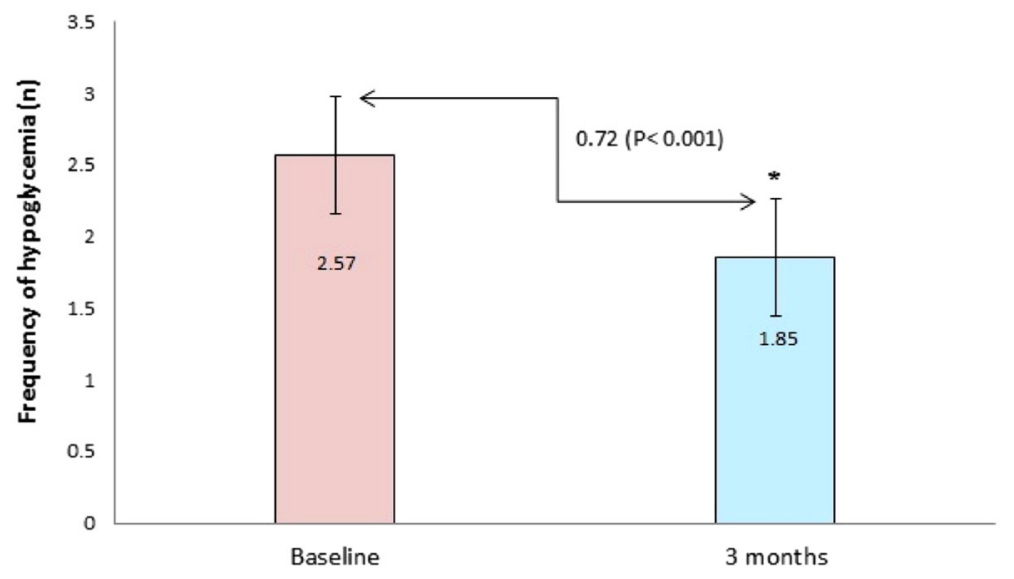

Fig. 2 The impact of professional continuous glucose monitoring on the frequency of hypoglycemia. Values are presented as mean \pm standard deviation. ${ }^{*}$ Baseline vs. 3 months compared by $t$ test

retrospective study, short-term blinded PCGM using the device in a private practice setting was reported to be ineffective for improving HbA1c levels in adult patients with type 1 and 2 diabetes [12]. Furthermore, the authors reported that before blinded CGM the average HbA1c level was $7.7 \pm 1.0 \%$ and after it was $7.8 \pm 1.1 \%$ [12]. A subgroup analysis of patients using continuous subcutaneous insulin infusion treatment also failed to show a significant HbA1c difference pre- and postCGM [12]. However, other studies support improvements in glycemic control using PCGM in different populations $[6,20,21]$.

Boland and colleagues [22] used PCGM in children with a mean HbA1c of $7.7 \%$ who were considered to have effectively controlled fasting glucoses. The PCGM showed over 90\% of patients had postprandial hyperglycemia, with almost half getting levels over $300 \mathrm{mg} / \mathrm{dL}$. Almost $70 \%$ of the CGMs revealed frequent and prolonged asymptomatic hypoglycemia (glucose $\quad<60 \mathrm{mg} / \mathrm{dL}$ ). The outcomes unquestionably disclosed that PCGM is relevant to improvement in diabetes management [22]. It should be noted here that real-time CGM was frequently removed by the patients prior than planned, mainly due to lag time between sensor and blood glucose, the need for calibration, skin irritation, technical problems, frequency of alarms, discomfort with sensor, cost and concerns with false detection of hypoglycemia, and failure to detect hypoglycemia compared with standard home monitoring [23]. However, the PCGM facilitate with no alarms, better convenience and minimum patient training compared with real-time CGM, and it allows users see the CGM data after it has been analyzed by a healthcare professional.

Hypoglycemia is a potentially fatal event, and people with T1DM describe severe hypoglycemia as the most frightening and feared event in their lives [24]. Studies have shown greater detection of hypoglycemia, and improved detection of severe hypoglycemia with the use of PCGM helps patients to reduce the number of episodes of hypoglycemia [2528]. In the present study, the authors found a positive decline in the frequency of hypoglycemia at 3 months in both male and female patients. Similar results in the frequency of hypoglycemia were observed in the different age groups and the different treatment groups. 
It should be noted here, that when compared to male patients, females showed a significant reduction in the frequency of hypoglycemia $(P<0.05)$. The current findings are in accordance with earlier studies which reported that PCGM practice decreases self-reported hypoglycemia [29].

The study has some limitations. Major limitations of this study include: a relatively small sample size; a limited number of risk factors examined; limited social and demographic factors; that it was performed at a single center; and that there was no control group with which to compare the study group. Therefore, results may not be generalizable to real-world situations. More studies on a larger scale are needed to address the limitations indicated in this study. Despite the limitations, the study delivers valuable data and creates awareness about the utility of CGM among patients with T1DM in Saudi Arabia.

\section{CONCLUSION}

While PCGM is a feasible preference for glycemic control, the use of PCGM should also be considered for patients with T1DM as it reduces HbA1c levels and the frequency of hypoglycemia. It is an initial step towards reaching the ideal diabetes management technology, one that is a closed-loop system.

\section{ACKNOWLEDGMENTS}

We would like to thank our patients for their participation in the study. No funding or sponsorship was received for this study or publication of this article. All named authors meet the International Committee of Medical Journal Editors (ICMJE) criteria for authorship for this manuscript, take responsibility for the integrity of the work as a whole, and have given final approval for the version to be published.

Conflict of interest. Ayman A. Al Hayek, Asirvatham A. Robert, Mohammed Al Dawish, Rania A. Ahmed, and Fahad S. Al Sabaan declare no conflict of interest.

Compliance with ethics guidelines. This study was conducted in accordance with the Helsinki Declaration of 1964, as revised in 2013, and the protocol of this study was approved by the research ethics committee of Prince Sultan Military Medical City, Riyadh, Saudi Arabia.

Open Access. This article is distributed under the terms of the Creative Commons Attribution Noncommercial License which permits any noncommercial use, distribution, and reproduction in any medium, provided the original author(s) and the source are credited.

\section{REFERENCES}

1. International Diabetes Federation. Diabetes Atlas. Brussels: International Diabetes Federation. 5th ed. 2011. http://www.idf.org/diabetesatlas/news/fifthedition-release. Accessed June 15, 2013.

2. Al-Hayek AA, Robert AA, Abbas HM, Itani MB, AlSaeed AH, Juhani AE, et al. Assessment of healthrelated quality of life among adolescents with type 1 diabetes mellitus in Saudi Arabia. Saudi Med J. 2014;35:712-7.

3. Alhowaish AK. Economic costs of diabetes in Saudi Arabia. J Fam Community Med. 2013;20:1-7.

4. Alqurashi KA, Aljabri KS, Bokhari SA. Prevalence of diabetes mellitus in a Saudi community. Ann Saudi Med. 2011;31:19-23.

5. Al-Hayek AA, Robert AA, Alzaid AA, Nusair HM, Zbaidi NS, Al-Eithan MH, et al. Association between diabetes self-care, medication adherence, anxiety, depression, and glycemic control in type 2 diabetes. Saudi Med J. 2012;33:681-3. 
6. Blevins TC. Professional continuous glucose monitoring in clinical practice 2010. J Diabetes Sci Technol. 2010;4:440-56.

7. Klonoff DC, Buckingham B, Christiansen JS, Montori VM, Tamborlane WV, Vigersky RA, et al. Continuous glucose monitoring: an Endocrine Society Clinical Practice Guideline. J Clin Endocrinol Metab. 2011;96:2968-79.

8. Phillip M, Danne T, Shalitin S, Buckingham B, Laffel L, Tamborlane $\mathrm{W}$, et al. Use of continuous glucose monitoring in children and adolescents $\left(^{*}\right)$. Pediatr Diabetes. 2012;13:215-28.

9. Mark R. Burge, Stephen Mitchell, Alison Sawyer, David S. Schade. Continuous glucose monitoring: the future of diabetes management. Diabetes Spectr. 2008;21(2):112-119. doi:10.2337/diaspect. 21.2.112.

10. Blevins TC, Bode BW, Garg SK, Grunberger G, Hirsch IB, Jovanovic L, et al. Statement by the American Association of Clinical Endocrinologists Consensus Panel on continuous glucose monitoring. Endocr Pract. 2010;16:730-45.

11. Juvenile Diabetes Research Foundation Continuous Glucose Monitoring Study G, Beck RW, Hirsch IB, Laffel L, Tamborlane WV, Bode BW, et al. The effect of continuous glucose monitoring in wellcontrolled type 1 diabetes. Diabetes Care. 2009;32:1378-83.

12. Pepper GM, Steinsapir J, Reynolds K. Effect of shortterm iPRO continuous glucose monitoring on hemoglobin A1c levels in clinical practice. Diabetes Technol Ther. 2012;14:654-7.

13. Irvine AA, Cox D, Gonder-Frederick L. Fear of hypoglycemia: relationship to physical and psychological symptoms in patients with insulindependent diabetes mellitus. Health Psychol. 1992;11:135-8.

14. American Diabetes Association. Standards of medical care in diabetes-2012. Diabetes Care. 2012;35(Suppl 1):S11-63. doi:10.2337/dc12-s011.

15. Al Hayek AA, Robert AA, Al Dawish MA, Braham RB, Goudeh HS, Al Sabaan FS. Efficacy of insulin pump therapy on diabetes treatment satisfaction and glycemic control among patients with type 1 diabetes mellitus in saudi arabia: a prospective study. Diabetes Ther. 2015. doi:10.1007/s13300015-0107-1.

16. De Block C, Manuel-y-Keenoy B, Van Gaal L. A review of current evidence with continuous glucose monitoring in patients with diabetes. J Diabetes Sci Technol. 2008;2:718-27.
17. Kovatchev BP. Diabetes technology: markers, monitoring, assessment, and control of blood glucose fluctuations in diabetes. Scientifica. 2012;2012:283821.

18. Lawlor MT, Laffel LM. New technologies and therapeutic approaches for the management of pediatric diabetes. Curr Diabetes Rep. 2001;1:56-66.

19. Larson NS, Pinsker JE. The role of continuous glucose monitoring in the care of children with type 1 diabetes. Int $\mathrm{J}$ Pediatr Endocrinol. 2013;2013:8.

20. Kaufman FR, Gibson LC, Halvorson M, Carpenter S, Fisher LK, Pitukcheewanont P. A pilot study of the continuous glucose monitoring system: clinical decisions and glycemic control after its use in pediatric type 1 diabetic subjects. Diabetes Care. 2001;24:2030-4.

21. Ludvigsson J, Hanas R. Continuous subcutaneous glucose monitoring improved metabolic control in pediatric patients with type 1 diabetes: a controlled crossover study. Pediatrics. 2003;111(5 Pt 1):933-8.

22. Boland E, Monsod T, Delucia M, Brandt CA, Fernando S, Tamborlane WV. Limitations of conventional methods of self-monitoring of blood glucose: lessons learned from 3 days of continuous glucose sensing in pediatric patients with type 1 diabetes. Diabetes Care. 2001;24:1858-62.

23. Secher AL, Madsen AB, Ringholm L, Barfred C, Stage E, Andersen HU, et al. Patient satisfaction and barriers to initiating real-time continuous glucose monitoring in early pregnancy in women with diabetes. Diabet Med. 2012;29:272-7.

24. Sanders K, Mills J, Martin FI, Del Horne DJ. Emotional attitudes in adult insulin-dependent diabetics. J Psychosom Res. 1975;19:241-6.

25. Chico A, Vidal-Rios P, Subira M, Novials A. The continuous glucose monitoring system is useful for detecting unrecognized hypoglycemias in patients with type 1 and type 2 diabetes but is not better than frequent capillary glucose measurements for improving metabolic control. Diabetes Care. 2003;26:1153-7.

26. Jensen $\mathrm{MH}$, Christensen TF, Tarnow L, Mahmoudi $\mathrm{Z}$, Johansen MD, Hejlesen OK. Professional continuous glucose monitoring in subjects with type 1 diabetes: retrospective hypoglycemia detection. J Diabetes Sci Technol. 2013;7:135-43.

27. Schiaffini R, Ciampalini P, Fierabracci A, Spera S, Borrelli P, Bottazzo GF, et al. The continuous glucose monitoring system (CGMS) in type 1 
diabetic children is the way to reduce hypoglycemic risk. Diabetes Metab Res Rev. 2002;18:324-9.

28. Wojciechowski P, Rys P, Lipowska A, Gaweska M, Malecki MT. Efficacy and safety comparison of continuous glucose monitoring and selfmonitoring of blood glucose in type 1 diabetes: systematic review and meta-analysis. Pol Arch Med Wewn. 2011;121:333-43.

29. Leinung M, Nardacci E, Patel N, Bettadahalli S, Paika $\mathrm{K}$, Thompson S. Benefits of short-term professional continuous glucose monitoring in clinical practice. Diabetes Technol Ther. 2013;15:744-7. 